\title{
Factor analysis of exercise capacity, dyspnoea ratings and lung function in patients with severe COPD
}

\author{
R.E. Wegner, R.A. Jörres, D.K. Kirsten, H. Magnussen
}

Factor analysis of exercise capacity, dyspnoea ratings and lung function in patients with severe COPD. R.E. Wegner, R.A. Jörres, D.K. Kirsten, H. Magnussen. CERS Journals Ltd 1994.

ABSTRACT: In this study we aimed to determine the relationship between exercise capacity, clinical ratings of dyspnoea and lung function parameters in patients with severe chronic obstructive pulmonary disease (COPD) by means of the statistical method of factor analysis.

Sixty two patients (mean age \pm SD, $66 \pm 9$ yrs) in stable clinical condition, with a forced expiratory volume in one second $\left(\mathrm{FEV}_{1}\right)<65 \%$ of predicted were investigated. Before the study, therapy was optimized, including inhaled bronchodilators, theophylline and steroids. Exercise capacity was determined from the best 6 min walking distance achieved in five self-paced treadmill walks performed on consecutive days. Lung function testing comprised spirometry and body plethysmography. Four different tools were chosen to rate dyspnoea and quality of life: the Baseline Dyspnoea Index (BDI), the Oxygen Cost Diagram (OCD), a modified Medical Research Council (MRC) Scale, and the Chronic Respiratory Disease Questionnaire (CRQ).

Principal component factor analysis revealed that the data could be reduced to three hypothetical underlying variables (factors), which accounted for $79 \%$ of the total variance. BDI, MRC, OCD, CRQ and walking distance were attributed to the first factor, forced expiratory volume in one second and airway resistance to the second factor, and lung volumes to the third factor.

Thus, our data suggest that the pathophysiological condition of severe COPD is characterized by three statistically independent entities: 1) exercise capacity, dyspnoea and quality of life ratings; 2) airway obstruction; and 3) pulmonary hyperinflation. These findings suggest that, as a physically measurable variable, the performance in a standardized walking test corresponds well to clinical ratings of dyspnoea and quality of life, whereas lung function parameters do not.

Eur Respir J., 1994, 7, 725-729.
Krankenhaus Grosshansdorf, Zentrum für Pneumologie und Thoraxchirurgie, LVA Freie und Hansestadt Hamburg, Federal Republic of Germany.

Correspondence: H. Magnussen

Krankenhaus Grosshansdorf Wöhrendamm 80

D - 22927 Grosshansdorf

Federal Republic of Germany

Keywords: Chronic airway obstruction pulmonary hyperinflation

quality of life

walking test

Received: July 201993

Accepted after revision November 211993
In patients with chronic obstructive pulmonary disease (COPD), various measures of outcome are used to assess the severity of disease, including parameters of pulmonary function, maximal respiratory pressures [1], exercise tolerance [2], and clinical ratings of dyspnoea [3, 4]. Among the functional impairments in patients with COPD, limitation of exercise tolerance is an essential feature which can be determined in a simple and standardized way as maximum walking distance within a fixed period of time [5-7].

To evaluate the overall pattern of functional impairments in COPD, the statistical method of factor analysis has recently been introduced [8, 9]. MAHLER and HARVER [9] found that spirometric parameters, maximal respiratory pressures, and dyspnoea scores represented three separate and independent statistical entities (factors). RIEs et al. [8], investigating a large set of variables, including gas exchange and symptoms at maximum exercise, in a broad range of patients with COPD, reported on four factors which comprised rather heterogeneous sets of variables and were interpreted as exercise tolerance, disease severity, lung volumes, and expiratory flow rates.

The aim of our study was to examine the relationship between exercise capacity, as assessed by a standardized walking test on the one hand, and lung function parameters and clinical ratings of dyspnoea on the other. Using a selected subset of parameters, we analysed only patients with severe COPD in order to reduce correlations which were not specific of the disease.

\section{Patients and methods}

\section{Patients}

We studied 62 patients with severe COPD, whose diagnosis was based on the guidelines of the American Thoracic 
Table 1. - Patient characteristics and squared multiple correlations (SMC) for each of the parameters included in the factor analysis

\begin{tabular}{lccc}
\hline Parameter & Mean \pm SD & Range & SMC \\
\hline Gender M/F & $51 / 11$ & - & - \\
Age yrs & $66 \pm 9$ & $47-79$ & - \\
Weight kg & $69 \pm 10$ & $40-90$ & - \\
Height cm & $171 \pm 8$ & $156-199$ & - \\
FEV $_{1} l$ \% & $1.1 \pm 0.4$ & $0.5-2.5$ & - \\
FEV ${ }_{1} \%$ pred & $39 \pm 13$ & $17-64$ & 0.6188 \\
VC l & $3.4 \pm 0.9$ & $1.9-5.6$ & - \\
VC \% pred & $90 \pm 16$ & $58-120$ & - \\
Raw cmH ${ }_{2} \mathrm{O} \cdot \mathrm{s} \cdot l^{-1}$ & $4.6 \pm 2.2$ & $1.4-12.2$ & 0.5169 \\
TGV l & $5.8 \pm 1.6$ & $2.9-9.8$ & - \\
TGV \% pred & $171 \pm 44$ & $86-303$ & 0.9223 \\
RV l \% & $4.6 \pm 1.4$ & $1.4-8.4$ & - \\
RV \% pred & $195 \pm 62$ & $55-392$ & 0.9329 \\
WD m & $434 \pm 158$ & $159-769$ & 0.4880 \\
CRQ score & $102 \pm 17$ & $59-129$ & 0.4883 \\
BDI score & $5.0 \pm 2.6$ & $0-11$ & 0.5512 \\
MRC score & $1.9 \pm 1.0$ & $0-4$ & 0.6636 \\
OCD mm & $54 \pm 15$ & $31-94$ & 0.6124 \\
\hline
\end{tabular}

VC: inspired vital capacity; $\mathrm{FEV}_{1}$ : forced expiratory volume in one second; Raw: airway resistance; TGV: thoracic gas volume; RV: residual volume; WD: walking distance; CRQ: Chronic Respiratory Disease Questionnaire; BDI: Baseline Dyspnoea Index; MRC: Medical Research Council scale; OCD: Oxygen Cost Diagram; \% pred: percentage predicted.

Society [10] (table 1). Patients were included on the basis of history of gradual progression of dyspnoea on exertion over many years, no history of atopy, forced expiratory volume in one second $\left(\mathrm{FEV}_{1}\right)$ of less than $65 \%$ of predicted, stable clinical condition, and absence of other major illnesses, such as congestive heart failure, which might interfere with exercise testing. Patients received theophylline as required to obtain trough serum theophylline levels of $8-20 \mathrm{mg} \cdot \mathrm{ml}^{-1}$ and, in addition, $4 \times 200 \mu \mathrm{g}$ of salbutamol or fenoterol and $4 \times 200 \mu \mathrm{g}$ of ipratropium bromide from metered dose inhalers, or equivalent doses in nebulized form. In those patients whose medication included systemic or inhaled steroids, no change was allowed for one week preceding or during the study. Predicted values for lung function parameters were calculated according to European Community for Coal and Steel (ECCS) [11]. All patients gave their informed consent, and the study protocol was approved by the Ethics Committee of the Medical Board of Schleswig-Holstein.

\section{Methods}

In each patient, exercise performance, lung function parameters, and clinical ratings of dyspnoea and quality of life were assessed during a five day period. Exercise performance was determined as the best value of five 6 min walking distances (WD) achieved in self-paced treadmill walking tests [7] performed on consecutive days. For this purpose, a horizontal treadmill (LE 2000, Jaeger, Würzburg, FRG) was supplied with a remote control module for the patient's use. Before each test, patients were instructed to walk as far as possible in 6 min, and no further encouragement was given during the test [6].

Lung function testing was performed on the fourth or fifth day, and comprised spirometry and body plethysmography. We measured $\mathrm{FEV}_{1}$, airway resistance (Raw), thoracic gas volume (TGV), and residual volume (RV) in a constant volume body plethysmograph (Bodytest, Jaeger, Würzburg, FRG).

Quality of life and overall dyspnoea were determined using the Chronic Respiratory Disease Questionnaire (CRQ) [12], the Baseline Dyspnoea Index (BDI) [13], a modified Medical Research Council (MRC) scale [14], and the Oxygen Cost Diagram (OCD) [15]. The CRQ is a disease-specific quality of life questionnaire, which assesses physical and emotional function. The BDI consists of five grades for the categories: functional impairment, magnitude of task, and magnitude of effort. The modified MRC scale includes five grades of various physical activities that provoke dyspnoea. The OCD is a visual analogue scale that corresponds to oxygen requirements of different activity levels. The CRQ and clinical rating scales of overall dyspnoea had been translated into German and were administered in a random order.

\section{Statistical analysis}

Mean values and standard deviations (SD) were computed for all parameters, and correlation among dyspnoea ratings and physiological parameters was assessed using Pearson's linear correlation coefficient [16]. To determine the degree of determination for each parameter, we computed its multiple correlation coefficient with the other parameters [16]. The matrix of Pearson's linear correlation coefficients was analysed by principal component analysis and varimax rotation [17]. The purpose of this procedure was to attribute the parameters to independent hypothetical factors, with the degree of dependence of each parameter on each factor being represented by a "factor loading". The rotation procedure aimed to maximize the loadings of each variable on one factor, whilst simultaneously minimizing the loadings on the other factors, separating the variables into distinct subgroups. Thus, in essence, factor analysis aims to bundle the parameters into independent subgroups, whereby, according to its mathematical concept, the common variation within each subgroup is derived from a hypothetical entity (factor). Mathematically, the approach chosen in this study (principal component analysis) results in a reduction of the multidimensional parameter space into one with fewer dimensions, the orthogonal axes of which are pointing into the directions of maximum data variation. These axes can be interpreted as representing the underlying factors. The number of factors is determined by a trade-off: it should be as small as possible, but large enough to explain most of the variation within the data. According to a common approach [17], we determined the number of factors from the number of eigenvalues [17] of the correlation matrix with magnitude $\geq 1$. 
Results

\section{Basic statistics}

Mean values, standard deviations and ranges for clinical ratings of dyspnoea and quality of life, exercise capacity and lung function parameters, as well as multiple correlation coefficients, are listed in table 1. All clinical rating scales of dyspnoea and quality of life correlated significantly with exercise performance (range of correlation coefficients, $0.41-0.63 ; \mathrm{p}<0.001)$ but showed weaker or no significant correlation $(-0.02-0.41)$ with lung function parameters (table 2).

Table 2. - Correlations between exercise capacity, clinical dyspnoea ratings, and lung function parameters (\% of predicted)

\begin{tabular}{lcccc}
\hline Parameter & BDI & CRQ & OCD & MRC \\
\hline FEV $_{1}$ & 0.34 & 0.32 & 0.30 & -0.34 \\
Raw & 0.25 & 0.41 & -0.29 & 0.32 \\
TGV & 0.04 & 0.02 & 0.08 & -0.03 \\
RV & -0.02 & 0.11 & 0.04 & 0.02 \\
WD & 0.54 & 0.41 & 0.52 & -0.63 \\
\hline
\end{tabular}

For abbreviations see legend to table 1.

\section{Principal component analysis}

The principal component analysis yielded three factors which accounted for $78.5 \%$ of the total variance of the data. BDI, MRC, OCD, CRQ and WD loaded predominantly on factor one, TGV and RV on factor two, whereas $\mathrm{FEV}_{1}$ and Raw loaded predominantly on factor three (table 3). When the analysis was repeated with absolute values of lung function parameters, the results did not, essentially, change. Similarly, results were not altered by dividing the total sample of patients on the basis of severity of airflow obstruction $\left(\mathrm{FEV}_{1}<\right.$ or $\geq$ $39 \%$ of predicted; each group containing $n=31$ patients) and repeating the analysis in both subgroups.

Table 3. - Rotated factor loadings

\begin{tabular}{lccc}
\hline & \multicolumn{3}{c}{ Factor } \\
\cline { 2 - 4 } Parameter & 1 & 2 & 3 \\
\hline MRC & -0.8800 & 0.0235 & -0.1415 \\
OCD & 0.8516 & 0.0298 & 0.1257 \\
BDI & 0.8346 & -0.0362 & 0.1032 \\
WD & 0.7502 & 0.1456 & 0.1035 \\
CRQ & 0.6730 & -0.0714 & 0.3271 \\
& 0.0173 & 0.9821 & -0.1020 \\
RV & 0.0524 & 0.9757 & 0.0028 \\
TGV & & & \\
& -0.1995 & -0.1326 & -0.9220 \\
Raw & 0.2408 & -0.4106 & 0.7712 \\
FEV & & & \\
\hline
\end{tabular}

For abbreviations see legend to table 1 .

\section{Discussion}

In the present study, we compared various parameters of lung function, clinical ratings of dyspnoea and quality of life, and walking distance in patients with severe COPD. Using factor analysis, we found that exercise capacity and clinical ratings, airway obstruction, and pulmonary hyperinflation were three separate and independent entities.

Several parameters have been used to assess the severity of disease in patients with COPD and to evaluate the effect of treatment. These measures comprise parameters of lung function and gas exchange, exercise performance, maximal respiratory pressures, and clinical ratings of dyspnoea and quality of life. Determination of walking distance in a standardized manner seems to be a simple method $[5,7]$ to assess the limitations of exercise capacity in patients with severe COPD, who are often unable to perform bicycle ergometer tests. In addition, such a walking test seems to be realistic for describing patients' limitations in day-to-day activities. Therefore, we have used a standardized version of this test. However, due to learning effects, the test requires that the patient is familiarized with the procedure by performing at least five walks [18]. The learning effect was also observed in our study, with the improvement in mean walking distance changing from $15 \%$ between the first two tests to $4 \%$ between the last two tests. For analysis, we have selected the best one of these five tests. However, it is noteworthy that the results of our statistical analysis were virtually unchanged when we included the second test instead of the best one.

Due to their mutual correlations, different functional parameters and clinical scores do not represent independent information. In several studies, the relationships between subsets of functional variables have been evaluated $[4,13,15,19-21]$. It has been shown that pulmonary function measurements can predict exercise capacity with some accuracy, provided that a broad range of disease severity is investigated or a normal group is included [19]. However, these relationships are less clear if the subgroup of patients with severe COPD is studied [19].

Two studies have used the statistical method of factor analysis to reveal the relationship between functional parameters in COPD [8, 9]. Our results confirm that factor analysis is suitable for this purpose, since three factors were sufficient to explain more than $75 \%$ of the total variance for 11 variables. In a group of COPD patients, MAHLER and HARVER [9] used factor analysis in order to demonstrate that clinical ratings of breathlessness and physiological function are separate dimensions underlying the pathophysiology of the disease. They evaluated nine variables including age and gender, spirometric parameters, maximal inspiratory and expiratory pressures, and three clinical ratings of dyspnoea. Spirometric parameters, maximal pressures and dyspnoea scores were attributed to three separate and independent factors. These authors did not include a measure of exercise capacity into their analysis. RIES et al. [8] investigated a set of 28 parameters, including pulmonary function, maximal 
inspiratory pressure, gas exchange during rest and exercise, two ratings of perceived symptoms at maximum exercise, as well as age and height. Their aim was to reduce the number of functional parameters, and thereby avoid unwanted redundancies. They found four meaningful factors, which they interpreted as exercise tolerance, disease severity, lung volumes, and expiratory flow rates. However, their entity "exercise tolerance" seems to comprise a rather heterogeneous set of variables. The same holds true for the factors "disease severity" and "expiratory flow rates"; for example, $\mathrm{FEV}_{1}$ loaded predominantly on "disease severity", whereas $\mathrm{FEV}_{1}$ as \% of predicted loaded on "expiratory flow rates". Their study did not demonstrate clearcut separation between factors, because several variables loaded significantly on more than one factor. Of course, this problem has been favoured by the large number of unselected variables.

In contrast, we have restricted the set of variables to those which have been shown to be meaningful in a previous study on the effects of theophylline therapy withdrawal, in which 38 of our patients with severe COPD had participated [22]. Thus, it appears that the parameters chosen in the present study are related to the state of the disease as controlled by therapeutic interventions [4]. In addition, we have eliminated age, height, and gender as explicit variables in our factor analysis, by referring the lung function parameters to their respective reference values.

It is noteworthy, that our factor "pulmonary hyperinflation" is clearly separated from the two other factors as indicated by its loadings (table 3 ). The same result becomes evident from the data of RIES et al. [8], indicating that in patients with COPD hyperinflation is an independent characteristic of the disease state and not directly linked to airway obstruction as reflected by airway resistance. Airway resistance and $\mathrm{FEV}_{1}$ were attributed to one easily interpretable factor, which we named "airway obstruction", and which was called "disease severity" by RIEs et al. [8]. Interestingly, the $\mathrm{FEV}_{1} \%$ of predicted loaded on a different factor in their study, whereas in our analysis results did not differ when either or both of these parameters were included. Thus, our analysis was not sensitive to different reference frames of these physiological parameters. We made a further attempt to validate the stability of the estimated factor pattern by including serum protein and albumin levels, which were considered not to depend on nutritional state and muscle mass in our COPD patients. These two parameters were found to represent a fourth factor, which was truly independent of the other three factors and did not disturb their loadings. Therefore, our analysis appeared to be fairly stable even if additional parameters were taken into account.

In contrast to MAHLER and HARVER [9], we have included airway resistance and lung volumes, as measured by body plethysmography, to quantify lung function. Parameters of airway obstruction were not associated with clinical ratings and walking distance, and the same was true for lung volume parameters. Therefore, we suggest that in severe COPD the limitation of exercise capacity is essentially not due to the airway obstruction or pulmonary hyperinflation per se. These findings are in accordance with the clinical observation that the impairment in lung function is a weak predictor of dyspnoea and exercise capacity in severe COPD $[15,19,21]$. The relative independence of exercise performance is underlined in our data by the fact that the percentage of variance of walking distance accounted for by the three factors was lower than for other variables.

MAhler and Harver [9], as well as Ries et al. [8], investigated a broad range of patients with COPD, with some of them showing even normal lung function values. We studied a more homogeneous group of patients with severe COPD, as reflected by the fact that all patients except one showed an $\mathrm{FEV}_{1} \leq 60 \%$ of predicted. By this restriction, we tried to avoid those correlations which could be produced by including normal or nearly normal subjects [19], and to focus on the characteristics of severe COPD. Since the validity of the correlation and factor analysis is based on the assumption of linearity, we checked the relationships between parameters by multiple scatter plots, and we found no indication of nonlinearities. In addition, the distributions of all parameters were symmetric, and median values were virtually identical with mean values. To check further for inhomogeneities within our data set, we separately analyzed those patients with an $\mathrm{FEV}_{1}<39 \%$ of predicted and those with an $\mathrm{FEV}_{1} \geq 39 \%$, which is the median value of our group. There were no major differences in the factor pattern between both subgroups or between the subgroups and the total group. In addition, these data indicate that our results were not influenced by the fact that the analysis was based on no more than 62 patients.

In conclusion, our data suggest that there are three separate entities which independently characterize the pathophysiological condition of severe COPD: 1) exercise capacity and ratings of dyspnoea and quality of life; 2) airway obstruction; and 3) pulmonary hyperinflation. The fact that walking distance and clinical ratings of dyspnoea and quality of life were attributed to one common factor suggests that, as a physically measurable variable, the performance in a standardized walking test corresponds best to these clinical scores. Our results underline the usefulness of questionnaires and walking tests in the evaluation of patients with severe COPD.

\section{References}

1. Bruschi C, Cerveri I, Zoia MC, et al. Reference values of maximal respiratory mouth pressures: a populationbased study. Am Rev Respir Dis 1992; 146: 790-793.

2. McGavin CR, Artvinli M, Naoe H, McHardy GJR. Twelve-minute walking test for assessing disability in chronic bronchitis. Br Med J 1976; 2: 822-823.

3. Mahler DA, Wells CK. Evaluation of clinical methods for rating dyspnea. Chest 1988; 93: 580-586.

4. Guyatt GH, Townsend M, Keller J, Singer N, Nogradi S. Measuring functional status in chronic lung disease: conclusions from a randomized control trial. Respir Med 1991; 85 (Suppl. B): 17-21.

5. Butland RJA. Two-, six- and 12-minute walking tests in respiratory disease. $\mathrm{Br}$ Med J 1982; 284: 1607-1608. 
6. Guyatt GH, Pugsley SO, Sullivan MJ, et al. Effect of encouragement on walking test performance. Thorax 1984; 39: 818-822.

7. Beaumont A, Cockroft A, Guz A. A self-paced treadmill walking test for breathless patients. Thorax 1985; 40: 459-464.

8. Ries AL, Kaplan RM, Blumberg E. Use of factor analysis to consolidate multiple outcome measures in chronic obstructive pulmonary disease. J Clin Epidemiol 1991; 44: 497-503.

9. Mahler DA, Harver A. A factor analysis of dyspnea ratings, respiratory muscle strength and lung function in patients with chronic obstructive pulmonary disease. Am Rev Respir Dis 1992; 145: 467-470.

10. American Thoracic Society. Standards for the diagnosis and care of patients with chronic obstructive pulmonary disease (COPD) and asthma. Am Rev Respir Dis 1987; 136: 225-244.

11. Quanjer PH. Standardized lung function testing. Bull Eur Physiopathol Respir 1983; 5 (Suppl.): 1-95.

12. Guyatt GH, Berman LB, Townsend M, Pugsley SO, Chambers LW. A measure of quality of life for clinical trials in chronic lung disease. Thorax 1987; 42: 773-778.

13. Mahler DA, Weinberg DH, Wells CK, Feinstein AR. The measurement of dyspnea. Contents, interobserver agreement, and physiologic correlates of two new clinical indexes. Chest 1984; 85: 751-758.

14. Mahler DA, Rosiello RA, Harver A, et al. Comparison of clinical dyspnea ratings and psychophysical measure- ments of respiratory sensation in obstructive airway disease. Am Rev Respir Dis 1987; 135: 1229-1233.

15. McGavin CR, Artvinli M, Naoe H, McHardy GJR. Dyspnoea, disability, and distance walked: comparison of estimates of exercise performance in respiratory disease. $B r$ Med J 1978; 2: 241-243.

16. Hartung J, Elpelt B, Kloesener KH. Statistik. Lehrund Handbuch der angewandten Statistik. MünchenWien, Oldenbourg Verlag, 1985.

17. Mardia KV, Kent JT, Bibby JM. Multivariate analysis. London, Academic Press, 1979.

18. Knox AJ, Morrison JFJ, Muers MF. Reproducibility of walking test results in chronic obstructive airways disease. Thorax 1988; 43: 388-392.

19. Pineda H, Haas F, Axen K, Haas A. Accuracy of pulmonary function tests in predicting exercise tolerance in chronic obstructive pulmonary disease. Chest 1984; 86 : 564-567.

20. Wolkove N, Dajczman E, Coacone A, Kreisman H. The relationship between pulmonary function and dyspnea in obstructive lung disease. Chest 1989; 96: 1247-1251.

21. Hay JG, Stone P, Carter J, et al. Bronchodilator reversibility, exercise performance and breathlessness in stable chronic obstructive pulmonary disease. Eur Respir $J$ 1992; 5: 659-664.

22. Kirsten DK, Wegner RE, Jörres RA, Magnussen H. Effects of theophylline therapy withdrawal in severe chronic obstructive pulmonary disease. Chest 1993; 104: 1101-1107. 